\title{
Registro de Galictis vittata (Carnivora: Mustelidae) en un área suburbana en el estado de Tabasco, México
}

\author{
Rodrigo García-Morales*(i), Brenda Berenice Diez de Bonilla-Cervantes² (iD \\ 1 Centro del Cambio Global y la Sustentabilidad, A.C. Centenario del Instituto Juárez S/N C.P. 86080, Villahermosa, México. \\ 2 Universidad Autónoma de Guadalajara, Campus Villahermosa. Prol. Paseo Usumacinta km 3.5, C.P. 86039, Villahermosa, \\ México. \\ *Correspondencia: r.garciamorales83@gmail.com
}

\section{Resumen}

El grisón (Galictis vittata) es una de las especies carnivoras menos estudiadas. Existe poca información sobre su presencia en el estado de Tabasco. En octubre de 2020 se observó un individuo de G. vittata en un área suburbana al sur de la ciudad de Villahermosa. El presente registro represente el primero de la especie en ambientes urbanizados. Estas áreas podrían ser importantes para la conservación de la especie en paisajes con influencia humana.

Palabras clave: amenazas, desarrollo urbano, grisón, uso de hábitat.

\begin{abstract}
The grison (Galictis vittata) is one of the least studied carnivorous species. There is little information about its presence in the state of Tabasco. In October 2020, an individual of G. vittata was observed in a suburban area south of the city of Villahermosa. This record represents the first of the species in urbanized environments. These areas could be important for the conservation of the species in landscapes with human influence.
\end{abstract}

Key words: threats, urban development, greater grison, habitat use.

El grisón (Galictis vittata) es un miembro de la familia Mustelidae; y es la única representante del género Galictis en México (Ramírez-Pulido et al. 2014). Es un animal solitario, de hábitos terrestres y principalmente diurno (Kaufmann \& Kaufmann 1965), aunque también puede estar activo durante la noche (Sunquist et al. 1989). Es carnívoro, se alimenta principalmente de pequeños roedores, aves, anfibios, reptiles y peces (Bisbal 1986; Sunquist et al. 1989). Por su alimentación desempeña un papel importante en la dinámica y estructura de las poblaciones de vertebrados en los ecosistemas en los que habita (Yensen \& Tarifa 2003; Roemer et al. 2009).

La especie se distribuye desde el sur de México, extendiéndose a través de Centroamérica, hasta el norte de Argentina y sur de Bolivia y Brasil (Yensen \& Tarifa 2003). En México se encuentra en las tierras bajas desde el sur de Tamaulipas, al este de San Luis Potosí, 
bajando por la costa del Golfo hasta Oaxaca, Chiapas y la Península de Yucatán (Chávez 2005; Contreras-Díaz et al. 2020). Se encuentra en una amplia variedad de hábitats terrestres que incluyen bosques tropicales perennifolios, subperenifolios, bosques tropicales semideciduos, bosques mesófilos de montaña, vegetación secundaria, vegetación ribereña, incluso se ha registrado en plantaciones y cultivos (Yensen \& Tarifa 2003; Chávez 2005; Contreras-Díaz et al. 2020).

Aunque la especie está incluida en los listados más recientes de mamíferos para el estado de Tabasco (Hidalgo-Mihart et al. 2016) existen pocos informes documentados de su presencia en gran parte del territorio estatal. A partir de una búsqueda de registros en bases de datos y literatura especializada se encontraron 12 registros (Tabla 1), de los cuales ocho corresponden a ejemplares reportados en el portal de ciencia ciudadana Naturalista de la Comisión Nacional para el Conocimiento y Uso de la Biodiversidad (Naturalista 2019); dos a ejemplares depositados en colecciones biológicas (VertNet 2019) y dos a observaciones en campo (Sánchez-Hernández et al. 2001; Sánchez-Hernández et al. 2005).

TABLA 1. Registros previos de grisón (Galictis vittata) en Tabasco, México. NE: no especificado

\begin{tabular}{llllc}
\hline \multicolumn{1}{c}{ Fuente } & Municipio & \multicolumn{1}{c}{ Localidad } & Año & Coordenadas \\
\hline VertNet & Centro & Villahermosa & 1959 & NE \\
VertNet & Balancán & NE & 1959 & NE \\
Sánchez-Hernández et al. 2001 & Huimanguillo & Carlos A. Madrazo & $1997-1998$ & $17.396450-93.662150$ \\
Sánchez-Hernández et al. 2005 & Tacotalpa & Tapijulapa & 1998 & NE \\
Naturalista & Balancán & NE & 2016 & $17.937995-91.349156$ \\
Naturalista & Centla & NE & 2016 & $18.529512-92.736292$ \\
Naturalista & Tacotalpa & NE & 2017 & $17.590548-92.820911$ \\
Naturalista & Paraíso & NE & 2019 & $18.461759-93.285933$ \\
Naturalista & Cárdenas & NE & 2019 & $18.14841-93.944764$ \\
Naturalista & Macuspana & NE & 2020 & $17.661159-92.424565$ \\
Naturalista & Balancán & NE & 2020 & $17.707096-91.443253$ \\
Naturalista & Balancán & NE & 2020 & $17.790782-91.597596$ \\
\hline
\end{tabular}

Las áreas suburbanas son un punto intermedio de un gradiente que va desde las zonas edificadas desprovistas de vegetación, hasta los ecosistemas naturales con poca intervención humana (McKinney 2002). En ellas, algunas especies de fauna silvestre pueden encontrar condiciones idóneas para su permanencia y protección en comparación con las áreas más urbanizadas (Morera-Chacón \& Ruiz-Chaves 2018; Angarita-Yanes \& Cáceres-Martínez 2019). Con el fin de aportar al conocimiento de la distribución de la especie en México, la presente nota describe un nuevo registro de G. vittata en un área suburbana en el estado de Tabasco, México.

El sitio de observación corresponde a un lote baldío, adyacente a un potrero abandonado de aproximadamente 17 hectáreas y rodeado por varias viviendas. La vegetación en los alrededores está conformada por pastos perennes, vegetación secundaria y árboles aislados característicos de zonas inundables. La temperatura media anual es de 27 a $28^{\circ} \mathrm{C}$ y un rango de precipitación que va de $50 \mathrm{~mm}$ en la época de secas hasta $350 \mathrm{~mm}$ en la época de lluvias (Aceves \& Rivera-Hernández 2019). El 12 de octubre de 2020, a las 10 h30 horas se registró la presencia de un individuo de G. vittata (Figura 1) en un área abierta parcialmente inundada en la ranchería Ixtacomitán 1era sección, al sur de la ciudad de Villahermosa; municipio de Centro, Tabasco, México; a una altitud de 10 msnm (17.962628, -92.972911 WGS 84; Figura 2). El individuo fue observado por aproximadamente 10 minutos durante los cuales permaneció olfateando el ambiente y observando el área; después de ello dio media vuelta y regreso por el camino por el que llegó. Durante el tiempo de observación no se logró determinar el sexo del ejemplar. 


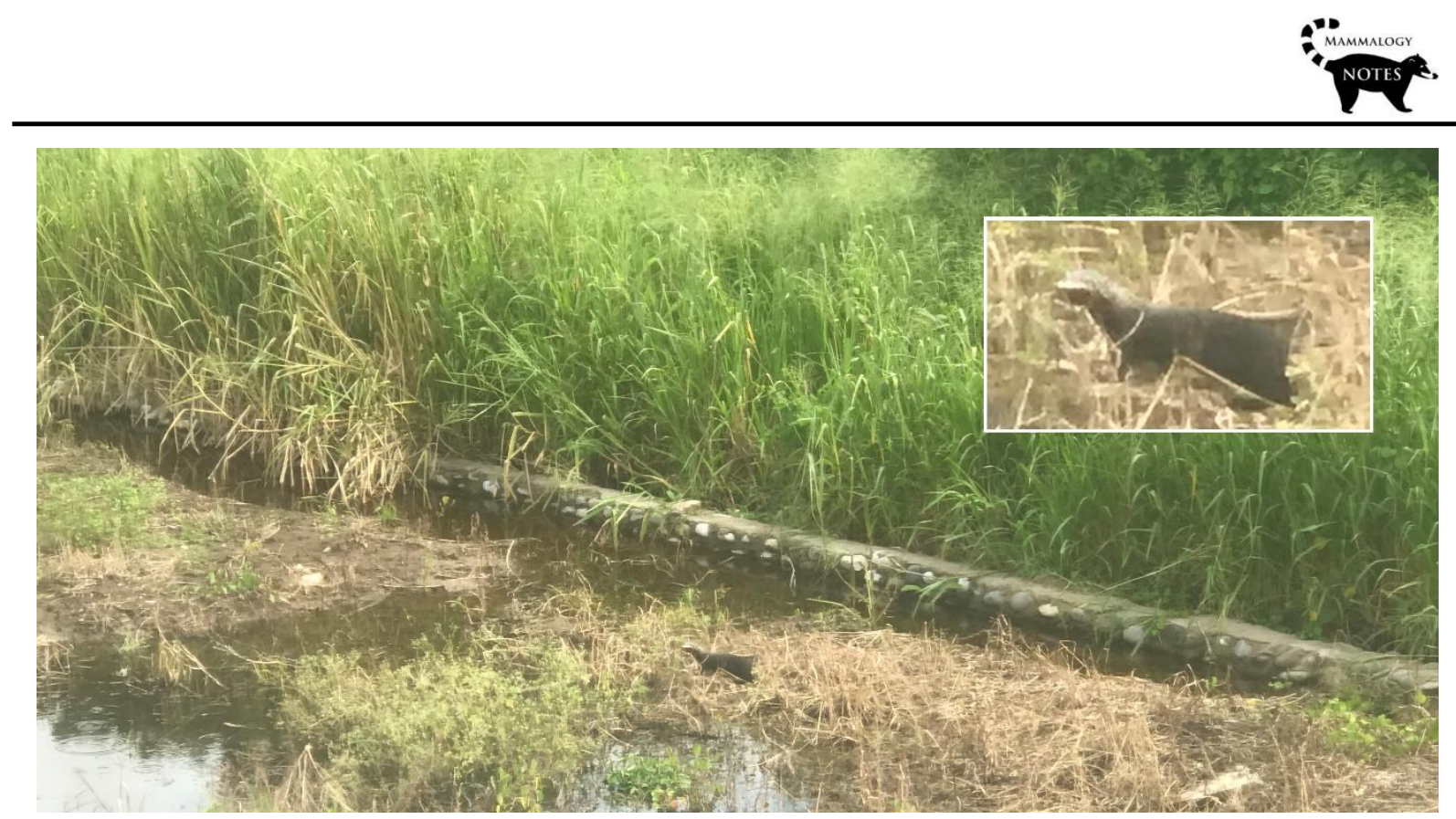

FIGURA 1. Individuo de Galictis vittata observado en la ranchería Ixtacomitán, Centro, Tabasco, México. Foto: Rodrigo García-Morales.

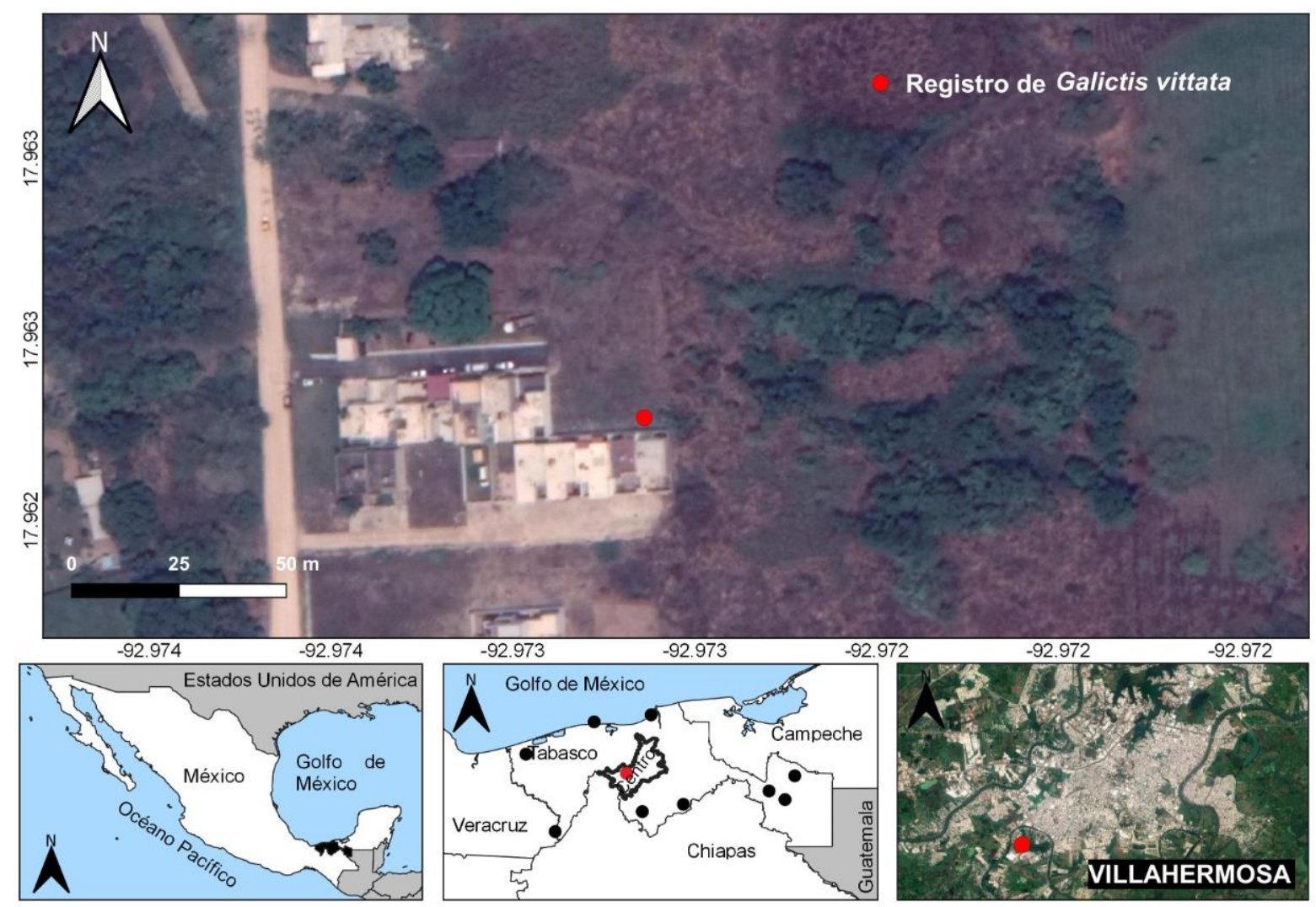

FIGURA 2. Ubicación geográfica del actual registro (punto rojo) y previos (puntos negros) de Galictis vittata en Tabasco, México.

La presente nota constituye el primer registro documentado de la presencia de la especie en un ambiente suburbano en México. Aunque existe un ejemplar colectado en la ciudad de Villahermosa en el año de 1959 (Tabla 1) el registro no cuenta con datos sobre las 
características ambientales al momento de la colecta por lo que no se podría afirmar que fue registrado en un ambiente urbano o suburbano. Las características ambientales del sitio (área abierta e inundada) coincide con lo mencionado por Yensen \& Tarifa (2003) como hábitats en los cuales puede estar presente la especie.

El sitio se encuentra rodeado por varias viviendas y fraccionamientos; además de dos carreteras con tránsito vehicular frecuente, por lo que las colisiones con automóviles podrían representar una amenaza para la especie en esta área. Los atropellamientos se encuentran entra las principales causas de mortandad de fauna silvestre que habita en áreas suburbanas o cerca de ellas (Delgado-Vélez 2014; Cupul-Magaña 2019). En México no existen datos de la frecuencia con que son atropellados. En Colombia, por ejemplo, suelen registrarte pocas muertes por colisión (Meza-Joya et al. 2019). Sin embargo, es importante mencionar que el $66 \%$ de los registros en Tabasco (Tabla 1) corresponden a ejemplares arrollados en las diferentes carreteras del estado.

Los incendios también podrían representar un peligro para el grisón; durante el año el potrero abandonado se incendia con frecuencia. Desde el avistamiento y hasta el 30 de abril de 2021 han ocurrido tres incendios en el predio. Esta situación podría ocasionar que la especie se desplace hacia otro sitio como consecuencia de la modificación de la vegetación o en un caso más grave la muerte por contacto directo con el fuego o intoxicación con humo. Otra amenaza que puede enfrentar la especie son las enfermedades trasmitidas por animales domésticos. En el sitio es común observar perros callejeros deambular por el predio; los cuales podrían ser reservorios o vectores de enfermedades; entre ellas el moquillo canino (canine distemper), la cual representa un riesgo para la especie en estos ambientes (Deem et al. 2000).

El presente registro demuestra que la especie es capaz de usar las áreas suburbanas. Sin embargo, se deben realizar estudios que permitan profundizar en el conocimiento de la biología y ecología del grisón en estos ambientes con el fin de implementar medidas de monitoreo y protección adecuadas.

\section{AGRADECIMIENTOS}

A Itzel Márquez López por su apoyo en la elaboración del mapa de ubicación geográfica y a Elizabeth Cristina Guedez Mikly por la edición de la figura 1.

\section{REFERENCIAS}

Aceves Navarro LA, Rivera-Hernández B. 2019. Clima. In: Comisión Nacional para el Conocimiento y Uso de la Biodiversidad, editor. La Biodiversidad en Tabasco. Estudio de estado. Vol 1. Distrito Federal, MEX: CONABIO press. p. 61-68.

Angarita-Yanes CE, Cáceres-Martínez CH. 2019. Reportes de felinos silvestres (Carnívora: Felidae) en el área urbana de Cúcuta, Nororiente de Colombia. Mammalogy Notes 5: 2-5. https://doi.org/10.47603/manovol5n2.2-5

Bisbal EFJ. 1986. Food habits of some Neotropical carnivores in Venezuela (Mammalia, Carnivora). Mammalia 50:329-340.

Chávez TJC. 2005. Galictis vittata (Schreber, 1776). In: Ceballos G, Oliva G, editors. Los mamíferos silvestres de México. Distrito Federal, MEX: CONABIO, Fondo de Cultura Económica press. p. 378-379. 
Contreras-Díaz C, Soria-Díaz L, Astudillo-Sánchez CC, Domínguez-Vega H, Gómez-Ortiz Y, MartínezGarcía L. 2020. Expansion of distribution range of the greater grison (Galictis vittata) in México. Therya Notes 1:1-4. https:// doi.org/10.12933/therya_notes-20-1

Cupul-Magaña FG. 2019. Registro del atropellamiento de Herpailurus yagouaroundi (Carnivora: Felidae) en la zona suburbana de Puerto Vallarta, México. Mammalogy Notes 5: 16-19.

Delgado-Vélez CA. 2014. Adicciones al atropellamiento vehicular de mamíferos en la vía de El Escobedo, Envigado (Antioquia), Colombia. Revista EIA 11: 147-153. https:/ / doi.org/10.24050/ reia.v11i22.679

Deem SL, Spelman LH, Yates RA, Montali RJ. 2000. Canine distemper in terrestrial carnivores: a review. Journal of Zoo and Wildlife Medicine 31:441-451.

Hidalgo-Mihart MG, Contreras-Moreno FM, De la Cruz AJ, Jiménez-Domínguez D, Juárez-López R, Oporto-Peregrino S, Ávila-Flores R. 2016. Mamíferos del estado de Tabasco. In: Briones-Salas M, Hortelano-Moncada Y, Magaña-Cota G, Sánchez-Rojas G, Sosa-Escalante JE, editors. Riqueza y Conservación de los Mamíferos en México a Nivel Estatal. Ciudad de México, MEX: Instituto de Biología-UNAM, Asociación Mexicana de Mastozoología A. C. y Universidad de Guanajuato press. p. 441-472.

Kaufmann JH, Kaufmann A. 1965. Observations of the behavior of tayras and grisons. Zeitschrift für Säugetierkunde 30:146-155.

McKinney ML. 2002. Urbanization, Biodiversity and conservation. Bioscience 52: 883-890. https:// doi.org/10.1641/0006-3568(2002)052[0883:UBAC]2.0.CO;2

Meza-Joya FL, Ramos E, Cardona D. 2019. Spatio-temporal patterns of mammal road mortality in Middle Magdalena Valley, Colombia. Oecologia Australis 23:575-588. https:/ / doi.org/10.4257/ oeco.2019.2303.15

Morera-Chacón BH, Ruiz-Chaves Y. 2018. Presencia suburbana de Puma concolor (Carnivora: Felidae) en el cantón de San Ramón, Alajuela, Costa Rica. Mammalogy Notes 4: 13-14.

Naturalista. 2019. Portal de ciencia ciudadana de la Comisión Nacional para el Conocimiento y Uso de la Biodiversidad (CONABIO). http:// naturalista.mx/. Consultado en 15 abril 2021.

Ramírez-Pulido J, González-Ruíz N, Gardner AL, Arroyo-Cabrales J. 2014. List of recent land mammals of Mexico. Special Publications of the Museum of Texas Tech University 63:1-69.

Roemer GW, Gompper ME, Van Valkengurgh B. 2009. The ecological role of the mammalian mesocarnivore. Bioscience 59:165-173. https:// doi.org/10.1525/ bio.2009.59.2.9

Sánchez-Hernández C, Romero-Almaraz ML, Colín-Martínez H, García-Estrada C. 2001. Mamíferos de cuatro áreas con diferente grado de alteración en el sureste de México. Acta Zoológica Mexicana 84:35-48.

Sánchez-Hernández C, Romero-Almaraz ML, García-Estrada C. 2005. Mamíferos. In: Bueno J, Álvarez F, Santiago S editors. Biodiversidad del estado de Tabasco. Distrito Federal, MEX: Instituto de Biología-UNAM, CONABIO press. p. 283-303.

Sunquist ME, Sunquist F, Daneke DE. 1989. Ecological separation in a Venezuelan llanos carnivore community. In: Redford KH, Eisenberg JF, editors. Advances in Neotropical Mammalogy. Gainesville, Florida, USA: Sandhill Crane Press. p. 197-232.

VertNet. 2019. National Science Foundation: Where discoveries begin. http://www.vertnet.org/. Accessed on 05 March 2021.

Yensen E, Tarifa T. 2003. Galictis vittata. Mammalian Species. 727:1-3. https:// doi.org/10.1644/0.727.1

Editor: Diego J. Lizcano

Recibido: 2021-03-27

Revisado: 2021-04-12

Aceptado 2021-05-04

Publicado 2021-06-24 